\title{
Local Fusion Graphs for Symmetric Groups
}

Ballantyne, John J and Greer, Nicholas M and Rowley, Peter J

2012

MIMS EPrint: 2012.7

Manchester Institute for Mathematical Sciences

School of Mathematics

The University of Manchester

\footnotetext{
Reports available from: http://eprints.maths.manchester.ac.uk/

And by contacting: The MIMS Secretary

School of Mathematics

The University of Manchester

Manchester, M13 9PL, UK
} 


\title{
Local Fusion Graphs for Symmetric Groups
}

\author{
John Ballantyne, Nicholas Greer, Peter Rowley
}

\begin{abstract}
For a group $G, \pi$ a set of odd positive integers and $X$ a set of involutions of $G$ we define a graph $\mathcal{F}_{\pi}(G, X)$. This graph, called a $\pi$-local fusion graph, has vertex set $X$ with $x, y \in X$ joined by an edge provided $x \neq y$ and the order of $x y$ is in $\pi$. In this paper we investigate $\mathcal{F}_{\pi}(G, X)$ when $G$ is a finite symmetric group for various choices of $X$ and $\pi$.
\end{abstract}

\section{Introduction}

There is a long and rich history of conjuring up various types of important combinatorial structures from a group. For example Cayley graphs, constructed from a group together with a generating set for that group, have had a considerable presence in such areas as geometric group theory and the study of expander graphs [22]. While groups with a $B N$-pair (such as groups of Lie-type) via their parabolic subgroups give rise to buildings (see Chapter 15 of [15]). For a group $G$ and $X$ a subset of $G$ we have the commuting graph, $\mathcal{C}(G, X)$, whose vertices are the elements of $X$ with two distinct elements of $X$ adjacent whenever they commute (for recent work on commuting graphs see [7], [8], [9], [10], [11], [12], [13], [18] and [19]). Such graphs have had an important impact in the study of finite simple groups, the commuting graphs associated with the Fischer groups [20], which featured in their construction, being a prime example. Variations on this theme have also played a role - see for example the so called root 4subgroups of the Held group, on page 230 of [2]. For yet another variety of graph consult [14].

We now discuss a recent combinatorial structure of this genre. Suppose that $G$ is a group, $\pi$ is a set of positive integers and $X$ is a subset of $G$. The graph $\mathcal{C}_{\pi}(G, X)$ is defined to be the graph with $X$ as its vertex set and for $x, y \in X x$ and $y$ are adjacent if $x \neq y$ and the order of $x y$ is in $\pi$. We observe, as $x y$ and $y x$ are conjugate elements of $G$, that the graph $\mathcal{C}_{\pi}(G, X)$ is undirected. Further, we observe that $\mathcal{C}_{\{2\}}(G, X)$ when $X$ is a set of involutions in $G$ is exactly the commuting involution graph $\mathcal{C}(G, X)$. When the orders of the elements in $X$ are coprime to all the integers in $\pi$, we shall call $\mathcal{C}_{\pi}(G, X)$ a $\pi$-coprimality graph (or just coprimality graph if $\pi$ is understood).

An important type of coprimality graph arises when $X$ is a set of involutions. For $\pi$ a set of odd positive integers, we write $\mathcal{F}_{\pi}(G, X)$ instead of $\mathcal{C}_{\pi}(G, X)$, and refer to $\mathcal{F}_{\pi}(G, X)$ as the $\pi$-local fusion graph for $X$. In the case when $\pi$ consists of all odd positive integers, we just write $\mathcal{F}(G, X)$ instead of $\mathcal{F}_{\pi}(G, X)$, and call 
$\mathcal{F}(G, X)$ the local fusion graph for $X$. The name 'local fusion' comes from the fact that if $x=x_{0}, x_{1}, x_{2}, \ldots, x_{m}=y$ is a path in the graph $\mathcal{F}(G, X)$, then $g=g_{1} g_{2} \ldots g_{m}$ conjugates $x$ to $y$ where each $g_{i}, 1 \leqslant i \leqslant m$, is an element of the dihedral group $\left\langle g_{i-1}, g_{i}\right\rangle$. In [17] $\{3\}$-local fusion graphs, $\mathcal{F}_{\{3\}}(G, X)$ are investigated for $X$ a $G$-conjugacy class of involutions. There issues, such as connectedness and what kind of triangles the graph contains, are examined. Further, the case when $G \cong P S L_{2}(q)$ ( $q$ a prime power) is analysed in detail, the work in [17] being prompted by a tower of graphs associated with a subgroup chain Alt(5) $\leq P S L_{2}(11) \leq M_{11} \leq M_{12}$. Each of the graphs in this tower may be viewed as being a restricted type of $\{3\}$-local fusion graph.

The famous Baer-Suzuki Theorem (see (39.6) in [1] or Theorem 3.8.2 in [21]), when $X$ is a $G$-conjugacy class of involutions, may be rephrased using the local fusion graph in the following way. The graph $\mathcal{F}(G, X)$ is totally disconnected if and only if $\langle X\rangle$ is a 2-subgroup of $G$. For suppose $\mathcal{F}(G, X)$ is totally disconnected, and let $x, y \in X$, with $x \neq y$. Assume that the order of $x y$ is $2^{k} m$, where $m$ is odd. If $m>1$, then $(x y)^{2^{k}}=x(y x \cdots x y)=x x^{g}$ has odd order $m$ and $x \neq x^{g}$. Hence $x$ and $x^{g}$ are adjacent in $\mathcal{F}(G, X)$, a contradiction. Therefore $x y$ has order $2^{k}$. Since, as is well known, $\langle x, y\rangle$ is a dihedral group of order twice that of $x y,\langle x, y\rangle$ is a 2-group, and so $\langle X\rangle$ is a 2-group by the Baer-Suzuki Theorem.

The aim of the present paper is to begin the investigation of $\pi$-local fusion graphs for finite symmetric groups.

Theorem 1.1. Suppose that $G=\operatorname{Sym}(n)$ with $n \geq 5$ and $X$ is a $G$-conjugacy class of involutions. Then $\mathcal{F}(G, X)$ is connected with $\operatorname{Diam}(\mathcal{F}(G, X))=2$.

For $n=2, \mathcal{F}(G, X)$ consists of just one vertex and for $n=3, \mathcal{F}(G, X)$ is the complete graph on 3 vertices. While for $n=4$ and $X$ the conjugacy class of $(1,2)(3,4)$ in $\operatorname{Sym}(4), \mathcal{F}(G, X)$ consists of three vertices with no edges - if $X$ is the conjugacy class of transpositions in $\operatorname{Sym}(4)$, then $\mathcal{F}(G, X)$ is connected of diameter 2 . There are $\pi$-local fusion graphs where we do encounter larger diameters. For example with $G=\operatorname{Sym}(9)$ and $X$ the $G$-conjugacy class of $(1,2)(3,4)(5,6)$ we have $\operatorname{Diam}\left(\mathcal{F}_{\{3\}}(G, X)\right)=\operatorname{Diam}\left(\mathcal{F}_{\{5\}}(G, X)\right)=$ $\operatorname{Diam}\left(\mathcal{F}_{\{7\}}(G, X)\right)=3$. This all prompts the question as to whether there are groups in which the diameter of local fusion graphs can be arbitrarily large - the answer is yes, and we direct the reader to [3]. For further work on coprimality graphs and symmetric groups see [5], and for more recent developments on local fusion graphs see [4] and [6].

The question of connectivity for $\pi$-local fusion graphs is the subject of our second theorem.

Theorem 1.2. Suppose that $G=\operatorname{Sym}(n), X$ is a $G$-conjugacy class of involutions and $\pi$ is a set of odd positive integers. Then $\mathcal{F}_{\pi}(G, X)$ is either totally disconnected or connected.

This paper is arranged as follows. Section 2 is mostly concerned with the notion of an ' $x$-graph' which, for $G \cong \operatorname{Sym}(n)$, encodes the $C_{G}(t)$-orbits on the 
conjugacy class of $t$ where $t$ is an involution. Then in Section 3 the $x$-graphs are put to work in establishing the diameter of local fusion graphs thereby proving Theorem 1.1. The proof of Theorem 1.2, which also employs $x$-graphs, is to be found in Section 4. Our group theoretic notation is standard as given, for example, in [1].

\section{Background Results}

Throughout this paper $t$ will denote a fixed involution of $X$, a conjugacy class of $\operatorname{Sym}(n)$. We will sometimes denote $\operatorname{Sym}(m)(m \in \mathbb{N})$ by $\operatorname{Sym}(\Omega)$ where $\Omega$ is an $m$-element set upon which the permutations act. For $g \in \operatorname{Sym}(\Omega)$, the support of $g, \operatorname{supp}(g)$, is $\Omega \backslash \operatorname{fix}(g)$, where $\operatorname{fix}(g)=\left\{\alpha \in \Omega \mid \alpha^{g}=\alpha\right\}$. We use $d(,$,$) to denote the standard graph theoretic distance on \mathcal{F}_{\pi}(G, X)$.

The proofs of Theorems 1.1 and 1.2 feature another graph $\mathcal{G}_{x}$ referred to as the $x$-graph. Assuming that $G=\operatorname{Sym}(n)$ acts upon $\Omega=\{1,2, \ldots, n\}$ and that $t=(1,2)(3,4) \ldots(2 b-1,2 b)$, we set

$$
\mathcal{V}=\{\{1,2\},\{3,4\}, \ldots,\{2 b-1,2 b\},\{2 b+1\}, \ldots,\{n\}\} .
$$

Thus the elements of $\mathcal{V}$ are just the orbits of $\langle t\rangle$ upon $\Omega$. For each $x \in X$, we define the $x$-graph $\mathcal{G}_{x}$ to be the graph with $\mathcal{V}$ as vertex set, and $v_{1}, v_{2} \in \mathcal{V}$ are joined by an edge whenever there exist $\alpha \in v_{1}$ and $\beta \in v_{2}$ with $\alpha \neq \beta$ for which $\{\alpha, \beta\}$ is a $\langle x\rangle$-orbit. Additionally the vertices of $\mathcal{G}_{x}$ corresponding to 2-cycles of $t$ will be coloured black $(\bullet)$ and the other vertices white (○). Therefore $\mathcal{G}_{x}$ has $b$ black vertices and $n-2 b$ white vertices. Note that the edges in $\mathcal{G}_{x}$ are in one-to-one correspondence with the 2-cycles of $x$. So the number of edges in $\mathcal{G}_{x}$ is the same as the number of black vertices. As an example, taking $n=16, t=(1,2)(3,4)(5,6)(7,8)(9,10)(11,12)$ and $x=$ $(1,3)(2,4)(5,6)(9,11)(12,13)(14,15), \mathcal{G}_{x}$ looks like

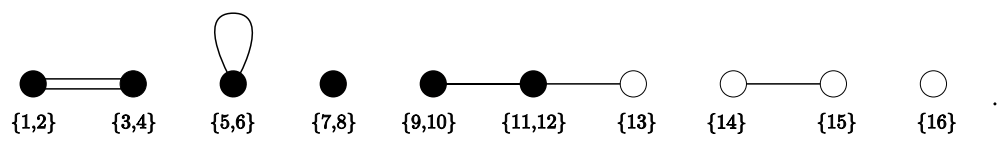

Lemma 2.1. For $x \in X$, the possible connected components of $\mathcal{G}_{x}$ are
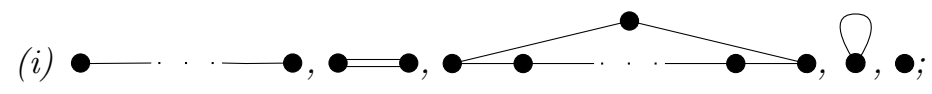

(ii)

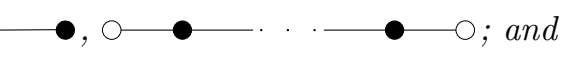

(iii) $\bigcirc \longrightarrow$, ○.

Proof. This follows from observing that a black vertex can have valency at most two while a white vertex has valency at most one.

Lemma 2.2. (i) Every graph with b black vertices of valency at most two, $n-2 b$ white vertices of valency at most one, and exactly $b$ edges is the $x$-graph for some $x \in X$. 
(ii) If $x, y \in X$, then $x$ and $y$ are in the same $C_{G}(t)$-orbit if and only if $\mathcal{G}_{x}$ and $\mathcal{G}_{y}$ are isomorphic graphs (where isomorphisms preserve the colour of vertices).

(iii) Let $C_{1}, C_{2}, \ldots, C_{\ell}$ be the connected components of $\mathcal{G}_{x}$. Assume that $x_{i}$ and $t_{i}$ are the corresponding parts of $x$ and $t$, and let $b_{i}, w_{i}$ and $c_{i}$ be, respectively, the number of black vertices, white vertices and cycles in $C_{i}$. Then

(a) the order of tx is the least common multiple of the orders of $t_{i} x_{i}$, $i=1, \ldots, \ell ;$ and

(b) for $i=1, \ldots, \ell$, the order of $t_{i} x_{i}$ is $\left(2 b_{i}+w_{i}\right) /\left(c_{i}+1\right)$.

Proof. See Lemma 2.1 and Proposition 2.2 of [7].

Suppose for $x \in X$ the connected components of $\mathcal{G}_{x}$ are $C_{1}, C_{2}, \ldots, C_{\ell}$, and for each such component let $x_{i}$ and $t_{i}$ be the corresponding parts of $x$ and $t$. Observe that for $i \neq j$ both $t_{i}$ and $x_{i}$ commute with both $t_{j}$ and $x_{j}$. So in the above example, $\ell=6$ with $t_{1}=(1,2)(3,4), t_{2}=(5,6), t_{3}=(7,8), t_{4}=$ $(9,10)(11,12)(13), t_{5}=(14)(15), t_{6}=(16)$, and $x_{1}=(1,3)(2,4), x_{2}=(5,6)$, $x_{3}=(7)(8), x_{4}=(9,11)(12,13)(10), x_{5}=(14,15), x_{6}=(16)$.

We remark that, as $\mathcal{G}_{x}$ has $b$ edges, the number of connected components of type $\bullet-\cdots$ and of type $\bigcirc \bullet-\cdots$ must be equal (including $\bigcirc \longrightarrow$ in the latter type). This is an important observation for part of the proof of Theorem 1.1. Consider the following simple situation: $t=$ $(1,2)(3,4)(5,6)(7)(8,9)(10)$ and $x=(1)(2,3)(4,5)(6)(7,8)(9,10)$, with $n=10$. Then $\mathcal{G}_{x}$ is
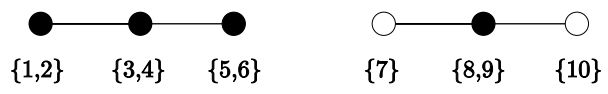

with $t_{1}=(1,2)(3,4)(5,6), t_{2}=(7)(8,9)(10), x_{1}=(1)(2,3)(4,5)(6)$ and $x_{2}=$ $(7,8)(9,10)$ being the parts of $t$ and $x$ corresponding to the two connected components. In our proof of Theorem 1.1 we argue by induction on $n$, and seek to exploit the symmetric subgroups $\operatorname{Sym}(\Lambda)$, where $\Lambda$ is the support of a connected part of $t$. But as we see in this small example, $t_{1}$ and $x_{1}$ are not conjugate in $\operatorname{Sym}(\{1,2,3,4,5,6\})$, nor are $t_{2}$ and $x_{2}$ in $\operatorname{Sym}(\{7,8,9,10\})$, and so our inductive strategy will fail. However, this obstacle may be overcome by

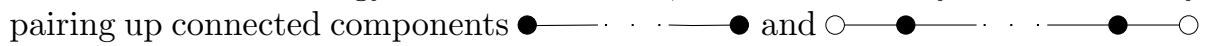
of $\mathcal{G}_{x}$ and applying induction to $\operatorname{Sym}(\Lambda)$ where $\Lambda$ is the union of the support of $t$ on these two connected components. This kind of issue does not arise with any of the other types of connected components of $\mathcal{G}_{x}$. While on the subject of potential pitfalls in the proof of Theorem 1.1 we mention the connected components $\longrightarrow$ of $\mathcal{G}_{x}$. Let $t_{i}$ and $x_{i}$ be the parts of $t$ and $x$ corresponding to this connected component, and set $\Lambda=\operatorname{supp}\left(t_{i}\right)$. Then $\operatorname{Sym}(\Lambda) \cong \operatorname{Sym}(4)$ with $t_{i}$ and $x_{i}$ having cycle type $2^{2}$ in $\operatorname{Sym}(\Lambda)$, and there is no path between $t_{i}$ and $x_{i}$ in the $\operatorname{Sym}(\Lambda)$ local fusion graph. To deal with such connected components of 
$\mathcal{G}_{x}$ we are forced to bring all of $\mathcal{G}_{x}$ into play - this turns out to be a substantial part of the proof of Theorem 1.1.

Suppose $x, y \in X$. We shall use $\mathcal{G}_{x}^{y}$ to denote the $x$-graph where $y$ plays the role of $t$ - so the vertices of $\mathcal{G}_{x}^{y}$ are the orbits of $\langle y\rangle$ on $\Omega$ with vertices $w_{1}, w_{2}$ joined if there exists $\alpha$ in $w_{1}$ and $\beta$ in $w_{2}$ with $\alpha \neq \beta$ and $\{\alpha, \beta\}$ an $\langle x\rangle$-orbit. So $\mathcal{G}_{x}^{t}$ is just $\mathcal{G}_{x}$. For more on $x$-graphs, see Section 2.1 of [7].

\section{The Diameter of $\mathcal{F}(G, X)$}

In this section we prove Theorem 1.1. So we have $G=\operatorname{Sym}(n)$ with $n \geq 5$, $X$ a $G$-conjugacy class of involutions and $t$ a fixed element of $X$. For $n \leq 16$, MAGMA [16] makes relatively short work of checking that $\mathcal{F}(G, X)$ is connected and has diameter 2 . So we may assume $n>16$.

We proceed by induction on $n$. Let $x \in X$. We aim to show that $d(t, x) \leq$ 2. Since there are plainly $x \in X$ for which $d(t, x)>1$, this would prove that $\operatorname{Diam}(\mathcal{F}(G, X))=2$. Suppose for the moment that $\mathcal{G}_{x}$ contains no connected components of type $\bullet$. If $\mathcal{G}_{x}$ is not connected and not of type $\bullet \bullet \bullet \quad \cdots$, then, by induction, $d(t, x) \leq$ 2. Thus, using Lemma 2.1, we may assume $\mathcal{G}_{x}$ is one of

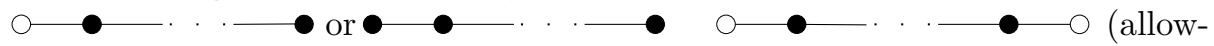
ing $\bigcirc \longrightarrow$ as a possibility in the latter component). In (3.1), (3.2) and (3.3) we deal with each of these possibilities in turn.

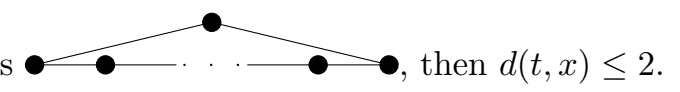

Assume, without loss of generality, that $t=(1,2)(3,4), \ldots,(2 m-1,2 m)$. So $\mathcal{G}_{x}$ has $m$ black vertices. If $m$ is odd, then $t x$ has odd order by Lemma 2.2(iii)(b), and so $d(t, x) \leq 1$. While if $m$ is even, we assume that $\mathcal{G}_{x}$ is labelled like so

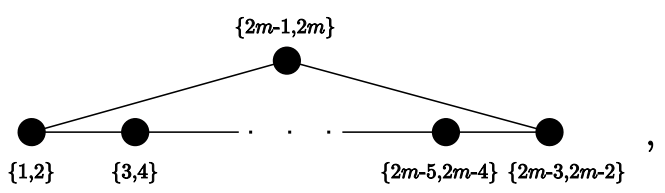

and that

$$
x=(1,2 m)(2,3)(4,5) \ldots(2 m-4,2 m-3)(2 m-2,2 m-1) .
$$

Note that we have $m \geq 4$. We select

$$
y=(1,2)(3,5)(4,2 m)(6,2 m-1)(7,8)(9,10) \ldots(2 m-3,2 m-2) .
$$

Then $y \in X$ and $t y=(3,2 m, 6)(4,5,2 m-1)$, and hence $d(t, y) \leq 1$. Now $\mathcal{G}_{x}^{y}$ is seen to be 


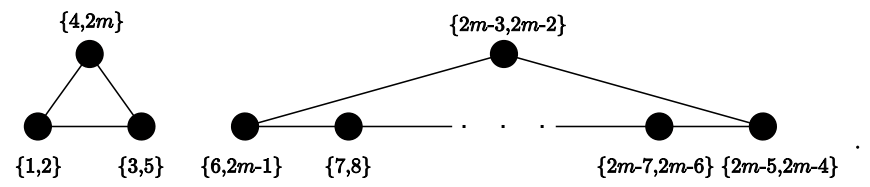

Since the two connected components of $\mathcal{G}_{x}^{y}$ have sizes 3 and $m-3$, both of which are odd, Lemma 2.2(iii) implies that $y x$ has odd order. Therefore $d(x, y) \leq 1$ and so (3.1) holds.

(3.2) If $\mathcal{G}_{x}$ is $\bigcirc \bullet \cdots$, , then $d(t, x)=1$.

Since $\mathcal{G}_{x}$ is a connected component with one white vertex, (3.2) follows from Lemma 2.2(iii).
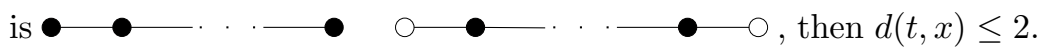

Without loss we may label $\mathcal{G}_{x}$ as follows
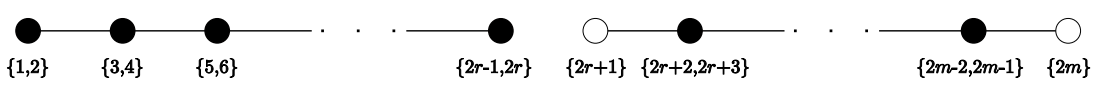

where

$t=(1,2)(3,4)(5,6) \ldots(2 r-1,2 r)(2 r+1)(2 r+2,2 r+3) \ldots(2 m-2,2 m-1)(2 m)$.

We may assume that

$$
x=(2,3)(4,5) \ldots(2 r-2,2 r-1)(2 r+1,2 r+2) \ldots(2 m-1,2 m) .
$$

Set $t_{0}=(1,2) t$ and $x_{0}=x(2 m-1,2 m)$. Then $t_{0}$ and $x_{0}$ are $H$-conjugate, where $H=\operatorname{Sym}\left(\Omega \backslash\{1,2 m\}\right.$ ). Observing that $\mathcal{G}_{x_{0}}^{t_{0}}$ (thinking of $t_{0}, x_{0}$ as involutions in $H$ ) has two connected components of type $\bigcirc-\cdots \longrightarrow$ we deduce from Lemma 2.2(iii) that $t_{0} x_{0}$ has odd order. Let $y=(1,2 m) t_{0}$. Then $y \in X$ and

$$
t y=(1,2) t_{0}(1,2 m) t_{0}=(1,2)(1,2 m)=(1,2,2 m),
$$

whence $d(t, y) \leq 1$. Also, as $t_{0}$ and $x_{0}$ fix 1 and $2 m$,

$$
\begin{aligned}
y x & =(1,2 m) t_{0} x_{0}(2 m-1,2 m) \\
& =t_{0} x_{0}(1,2 m)(2 m-1,2 m) \\
& =t_{0} x_{0}(1,2 m-1,2 m) .
\end{aligned}
$$

Now $t_{0} x_{0} \in H$ is a product of two disjoint odd cycles of lengths, say, $m_{1}, m_{2}$. If $2 m-1$ is in say the latter cycle of $t_{0} x_{0}$, then $t x$ is a disjoint product of an $m_{1}$-cycle and an $\left(m_{2}+2\right)$-cycle. Thus $y x$ has odd order and so $d(x, y) \leq 1$. Therefore $d(t, x) \leq 2$, which proves (3.3).

Taken together (3.1), (3.2) and (3.3) prove Theorem 1.1 when $\mathcal{G}_{x}$ contains no connected components of type $\bullet$. It therefore remains to analyse $\mathcal{G}_{x}$ when 
it has connected components of type $\longrightarrow$. If there are an even number of - connected components, then, as the local fusion graphs for $\operatorname{Sym}(8)$ have diameter two, by pairing them up and using induction we obtain our result. Thus we may assume $\mathcal{G}_{x}$ contains exactly one $\longrightarrow$ connected component. Let $\mathcal{H}_{x}$ denote the union of all the other connected components of $\mathcal{G}_{x}$. Also we may assume $t=(1,2)(3,4) t_{0}, x=(1,3)(2,4) x_{0}$ where $t_{0}$ and $x_{0}$ are involutions in $H=\operatorname{Sym}(\Omega \backslash\{1,2,3,4\})$.

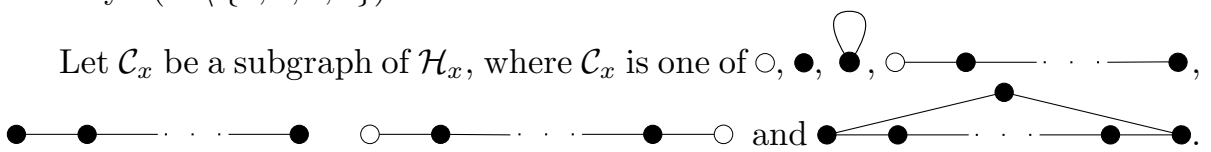

Then $t_{0}=t_{1} t_{2}, x_{0}=x_{1} x_{2}$ where $t_{1}, x_{1}$ are the parts in $\mathcal{C}_{x}$ and $t_{2}, x_{2}$ the parts in $\mathcal{H}_{x} \backslash \mathcal{C}_{x}$. Then $t_{2}$ and $x_{2}$ are conjugate involutions in some symmetric subgroup of $G$ and the $x_{2}$-graph (with respect to $t_{2}$ ) is $\mathcal{H}_{x} \backslash \mathcal{C}_{x}$. Since $\mathcal{H}_{x}$ contains no subgraph $\longrightarrow$ we can find $y_{2}$ in this conjugacy class such that $t_{2} y_{2}$ and $y_{2} x_{2}$ have odd order. Since $y_{2}$ commutes with both $(1,2)(3,4) t_{1}$ and $(1,3)(2,4) x_{1}$, without loss we may assume that $\mathcal{H}_{x}=\mathcal{C}_{x}$. We now work through the possibilities for $\mathcal{H}_{x}$ making repeated use of Lemma 2.2(iii) to show $d(t, x) \leq$ 2. The first three possibilities listed above do not need attention as $n \geq 16$.

If $\mathcal{H}_{x}$ is

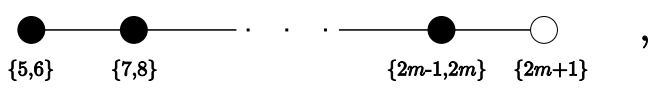

then

$$
t=(1,2)(3,4)(5,6)(7,8) \ldots(2 m-1,2 m)(2 m+1)
$$

and, without loss of generality,

$$
x=(1,3)(2,4)(5)(6,7)(8,9) \ldots(2 m, 2 m+1) .
$$

In the case when $m$ is odd, we select

$$
y=(1,5)(2,3)(4,2 m)(6,7)(8,9) \ldots(2 m-2,2 m-1)(2 m+1)
$$

and then $\mathcal{G}_{y}$ is

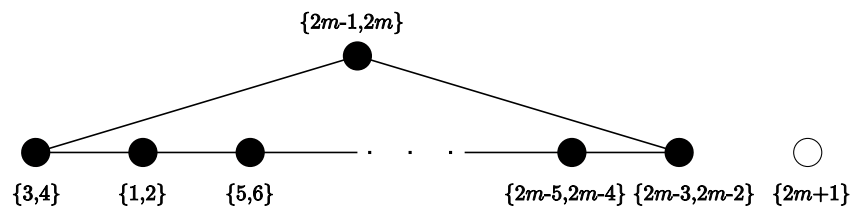

while $\mathcal{G}_{x}^{y}$ is

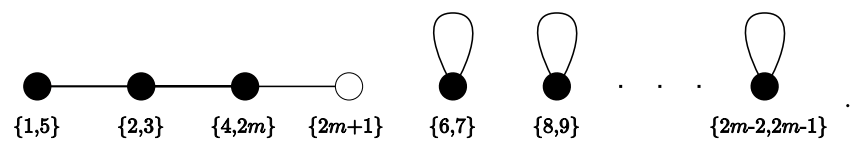


So $\mathcal{G}_{y}$ consists of a cycle of $m$ black vertices and one white vertex while $\mathcal{G}_{x}^{y}$ has one connected component with three black vertices and one white vertex with each of the other components being a cycle with one black vertex. Consequently ty and $y x$ both have odd order by Lemma 2.2 (iii), whence $d(t, x) \leq 2$. If $m$ were to be even, instead we choose

$$
y=(1,2 m-1)(2,6)(3,4)(5,2 m)(7,8)(9,10) \ldots(2 m-3,2 m-2)
$$

which gives $\mathcal{G}_{y}$ as

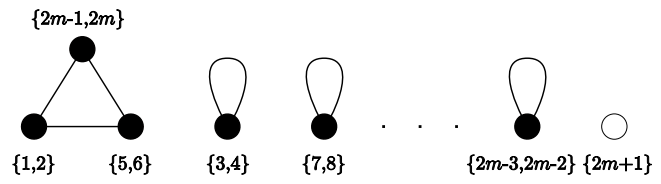

and $\mathcal{G}_{x}^{y}$ as

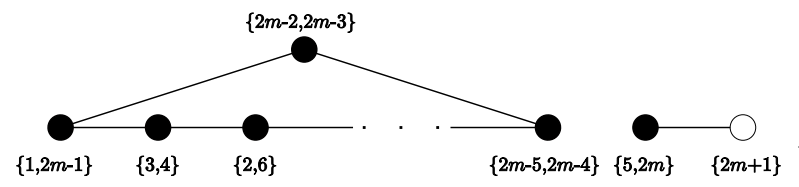

Here the cycle of black vertices in $\mathcal{G}_{x}^{y}$ has $m-1$ black vertices whence using Lemma 2.2(iii) again we deduce that $d(t, x) \leq 2$, and this settles the case when $\mathcal{H}_{x}$ is $\bigcirc$

Now we examine the case when $\mathcal{H}_{x}$ is

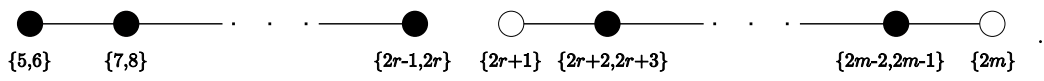

So

$t=(1,2)(3,4)(5,6) \ldots(2 r-1,2 r)(2 r+1)(2 r+2,2 r+3) \ldots(2 m-2,2 m-1)(2 m)$

and

$x=(1,3)(2,4)(5)(6,7)(8,9) \ldots(2 r-2,2 r-1)(2 r)(2 r+1,2 r+2) \ldots(2 m-1,2 m)$.

Choosing

$$
y=(1,2 m)(2,3)(4,5) \ldots(2 r-2,2 r-1)(2 r+1,2 r+2) \ldots(2 m-3,2 m-2),
$$

we observe that $\mathcal{G}_{y}$ is

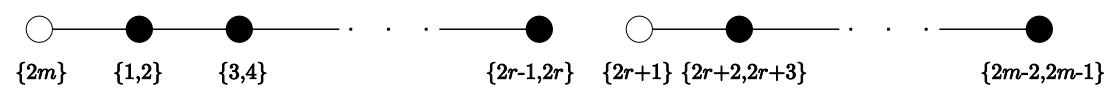

and $\mathcal{G}_{x}^{y}$ is
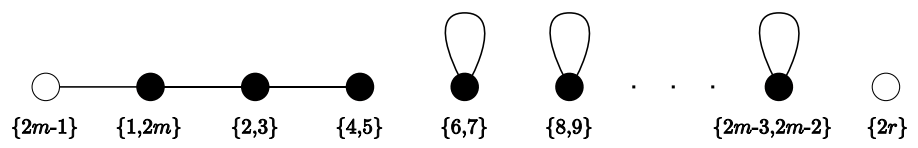
Yet again Lemma 2.2(iii) shows that $d(t, y) \leq 1 \geq d(y, x)$ so dealing with this possibility for $\mathcal{H}_{x}$.

We now consider our final case which is when $\mathcal{H}_{x}$ is

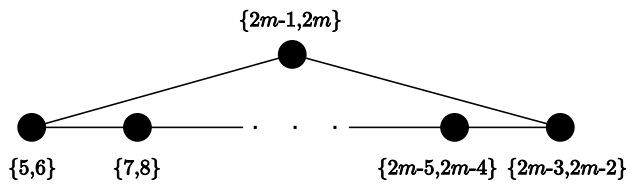

Thus

$$
t=(1,2)(3,4)(5,6)(7,8) \ldots(2 m-1,2 m)
$$

and, without loss,

$$
x=(1,3)(2,4)(6,7)(8,9) \ldots(2 m, 5) .
$$

When $m$ is even we select

$$
y=(1,5)(2,2 m)(3,4)(6,2 m-1)(7,8)(9,10) \ldots(2 m-3,2 m-2)
$$

and as a result $\mathcal{G}_{y}$ is

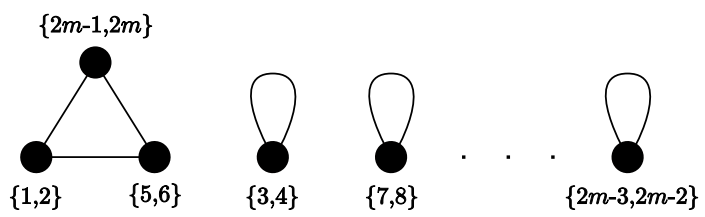

and $\mathcal{G}_{x}^{y}$ is

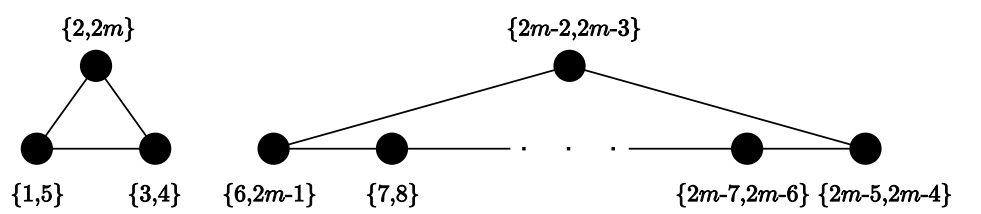

Before dealing with $m$ odd we recall that we are assuming $n(=2 m) \geq 16$. So $2 m-4>10$ and therefore the choice we now make gives us an element of $X$. Take

$$
\begin{gathered}
y=(1,2 m-4)(2,2 m)(3,4)(5,7)(6,9)(8,2 m-1)(10,11)(12,13) \ldots \\
\ldots(2 m-6,2 m-5)(2 m-3,2 m-2) .
\end{gathered}
$$

Hence $\mathcal{G}_{y}$ is

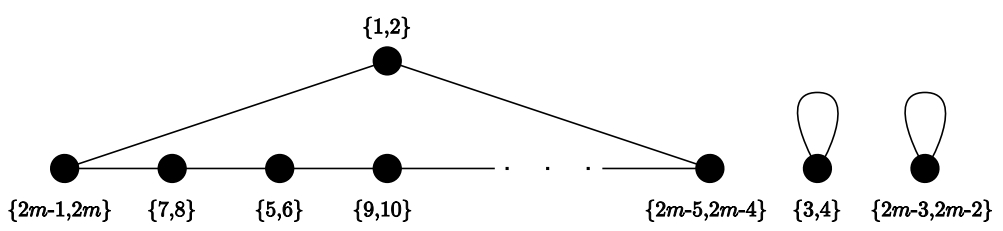


and $\mathcal{G}_{x}^{y}$ is

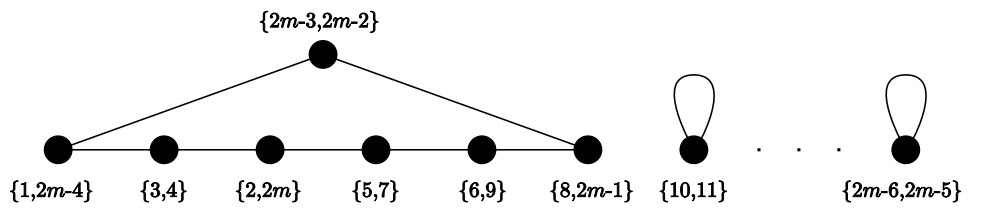

Use of Lemma 2.2(iii) shows that whether $m$ is even or odd we have $d(t, x) \leq 2$.

Having successfully dealt with all the possibilities for $\mathcal{H}_{x}$, the proof of Theorem 1.1 is complete.

\section{Connectedness of $\mathcal{F}_{\pi}(G, X)$}

As promised here we prove Theorem 1.2 which we restate.

Theorem 4.1. Suppose that $G=\operatorname{Sym}(n), X$ is a $G$-conjugacy class of involutions and $\pi$ is a set of odd positive integers. Then $\mathcal{F}_{\pi}(G, X)$ is either totally disconnected or connected.

Proof. We argue by induction on $n$, with $n=1$ clearly holding. Assume that $\mathcal{F}_{\pi}(G, X)$ is not totally disconnected, and let $t \in X$ be such that $Y$, the connected component of $t$ in $\mathcal{F}_{\pi}(G, X)$, has $|Y|>1$. Put $K=\operatorname{Stab}_{G}(Y)$. If $K=G$, then $Y=X$ and hence $\mathcal{F}_{\pi}(G, X)$ is connected. So we now suppose $K \neq G$, and argue for a contradiction.

Let $x \in Y$ with $d(t, x)=1$. Then $z=t x$ has order in the set $\pi$, and we have

(4.1) $\left\langle C_{G}(t), C_{G}(x)\right\rangle \leq K$, and

(4.2) $\operatorname{supp}(t) \cup \operatorname{supp}(x)=\Omega$.

If (4.2) is false, then $t$ and $x$ both fix some $\alpha \in \Omega$. So $t, x \in G_{\alpha} \cong \operatorname{Sym}(n-1)$. Since $t$ and $x$ are $G_{\alpha}$-conjugate and the order of $t x$ is in $\pi$, by induction $\mathcal{F}_{\pi}\left(G_{\alpha}, X \cap G_{\alpha}\right)$ is connected. Therefore $G_{\alpha} \leq K$, and so, as $K \neq G$ and $G_{\alpha}$ is a maximal subgroup of $G, K=G_{\alpha}$. If $t$ fixes a further element of $\Omega$, say $\beta$, then, by (4.1), $(\alpha, \beta) \in C_{G}(t) \leq K$, contrary to $K=G_{\alpha}$. So $t$ (and hence also $x$ ) fixes only $\alpha$. Thus $\mathcal{G}_{x}$ has only one white node (namely $\{\alpha\}$ ) with the remaining connected components being either

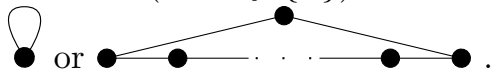
Without loss we assume $\alpha=n$.

Suppose that $\mathcal{G}_{x}$ has as a component. So, without loss of generality,

$$
t=(1,2)(3,4) \ldots(n-2, n-1)=(1,2) t_{1}
$$

and $x=(1,2) x_{1}$, where $x_{1} \in \operatorname{Sym}(\{3,4, \ldots, n-1\})$. Since $K \neq G$, we must have $n>3$. Thus $t_{1}, x_{1} \in H=\operatorname{Sym}(\{3,4, \ldots, n-1\})$ with $t_{1}$ and $x_{1}$ being $H$-conjugate involutions and the order of $t_{1} x_{1}$, being the same as that of $t x$, lies 
in $\pi$. Using induction again we infer that $\mathcal{F}_{\pi}\left(H, t_{1}^{H}\right)$ is connected. Hence, in $\mathcal{F}_{\pi}\left(H, t_{1}^{H}\right)$ there is a path from $t_{1}$ to

$$
s_{1}=(3,4)(5,6) \ldots(n-4, n-3)(n-1, n),
$$

say $t_{1}=y_{0}, y_{1}, \ldots, y_{m}=s_{1}\left(y_{i} \in t_{1}^{H}\right)$. Consequently

$$
t=(1,2) t_{1}=(1,2) y_{0},(1,2) y_{1}, \ldots,(1,2) y_{m}=(1,2) s_{1}
$$

is a path in $\mathcal{F}_{\pi}(G, X)$ from $t$ to

$$
(1,2)(3,4)(5,6) \ldots(n-4, n-3)(n-1, n) .
$$

But then $(n-1, n) \in K$, whereas $K=G_{\alpha}$. This rules out as being a connected component of $\mathcal{G}_{x}$.

Let $t=t_{1} t_{2} \cdots t_{k}$ and $x=x_{1} x_{2} \cdots x_{k}$, where

$$
\begin{aligned}
t_{1} & =(1,2) \ldots\left(\ell_{1}-1, \ell_{1}\right), \\
t_{2} & =\left(\ell_{1}+1, \ell_{1}+2\right) \ldots\left(\ell_{1}+\ell_{2}-1, \ell_{1}+\ell_{2}\right), \\
& \vdots
\end{aligned}
$$

and

$$
\begin{aligned}
& x_{1}=(2,3)(4,5) \ldots\left(\ell_{1}-2, \ell_{1}-1\right)\left(1, \ell_{1}\right), \\
& x_{2}=\left(\ell_{1}+2, \ell_{1}+3\right) \ldots\left(\ell_{1}+\ell_{2}-2, \ell_{1}+\ell_{2}-1\right)\left(\ell_{1}+1, \ell_{1}+\ell_{2}\right),
\end{aligned}
$$

So the elements $x_{1}, x_{2}, \ldots$ correspond to the connected components of $\mathcal{G}_{x}$. By Lemma 2.2(iii)(b) $t_{1} x_{1}$ has order $m=\ell_{1} / 2$. Now the order of $z=t x$ is the least common multiple of the orders of $t_{1} x_{1}, t_{2} x_{2}, \ldots, t_{k} x_{k}$, whence $m$ must be odd. Put

$$
w=(n, 1,3,5, \ldots, m-2, m-1, m-3, \ldots, 6,4,2) .
$$

Then $w$ is a cycle of length $m$, and so of order $m$. Further (by design) $w^{t_{1}}=w^{-1}$ and hence

$y_{1}=t_{1} w=(1, n)(2,3)(4,5) \ldots(m-3, m-2)(m, m+1)(m+2, m+3) \ldots\left(\ell_{1}-1, \ell_{1}\right)$

is conjugate to $t_{1}$. Also, of course, $t_{1} y_{1}=w$ has order $m$. So $y=y_{1} t_{2} \cdots t_{k} \in X$ and the order of $t y$ is the same as that of $t x$. Therefore $y \in Y$ and hence $(1, n) \in K$. This contradicts the earlier deduction that $K=G_{\alpha}$, and with this we have proven $(4.2)$.

(4.3) $K$ acts transitively and primitively on $\Omega$.

Since $C_{G}(t)$ and $C_{G}(x)$ have shape $2^{k} \operatorname{Sym}(2 k) \times \operatorname{Sym}(n-2 k)$, where $k=$ 
$|\operatorname{supp}(t)| / 2$, and $t$ and $x$ do not commute, (4.1) and (4.2) imply that $K$ is transitive on $\Omega$. Suppose $K$ does not act primitively on $\Omega$. Then we may choose a nontrivial block $\Lambda$ for $K$ with $\alpha \in \Lambda \cap \operatorname{supp}(t)$. If $\Lambda \nsubseteq \operatorname{supp}(t)$, then the action of $C_{G}(t)$ on $\Omega$ results in $\Lambda=\Omega$. Thus $\Lambda \subseteq \operatorname{supp}(t)$. Again, using the action of $C_{G}(t)$ on $\Omega$ we deduce that either $\Lambda=\operatorname{supp}(t)$ or $\Lambda=\{\alpha, \beta\}$ where $\beta=\alpha^{t}$. Since $t$ and $x$ do not commute, we may further assume that $\alpha \in \operatorname{supp}(x)$ is such that $\alpha^{x} \notin\{\alpha, \beta\}$. So $\alpha \in \operatorname{supp}(x)$ and a similar argument yields that either $\Lambda=\operatorname{supp}(x)$ or $\Lambda=\left\{\alpha, \alpha^{x}\right\}$. In view of (4.2) this then implies that $\Lambda=\Omega$, contrary to $\Lambda$ being a nontrivial block. Thus (4.3) holds.

Plainly $C_{G}(t)$, and hence $K$, contains transpositions. Thus Jordan's theorem [23] and (4.3) force $K=G$. With this contradiction the proof of the theorem is complete.

\section{References}

[1] M. Aschbacher: Finite group theory, Second edition. Cambridge Studies in Advanced Mathematics, 10. Cambridge University Press, Cambridge, 2000.

[2] M. Aschbacher: Sporadic groups, Cambridge Tracts in Mathematics, 104. Cambridge University Press, Cambridge, 1994.

[3] J. Ballantyne: On Local Fusion Graphs of Finite Coxeter Groups, MIMS Eprint 2011.2, Manchester Institute for Mathematical Sciences, The University of Manchester, UK.

[4] J. Ballantyne: Local Fusion Graphs of Finite Groups, PhD. Thesis, University of Manchester, 2011.

[5] J. Ballantyne, N. Greer, P. Rowley: On Coprimality Graphs for Symmetric Groups, MIMS Eprint 2010.64, Manchester Institute for Mathematical Sciences, The University of Manchester, UK. .

[6] J. Ballantyne, P. Rowley: A Note on Computing Involution Centralizers, MIMS Eprint 2011.32, Manchester Institute for Mathematical Sciences, The University of Manchester, UK.

[7] C. Bates, D. Bundy, S. Perkins, P. Rowley: Commuting Involution Graphs for Symmetric Groups, J. Algebra 266 (2003), 133-153.

[8] C. Bates, D. Bundy, S. Perkins, P. Rowley: Commuting Involution Graphs for Finite Coxeter Groups, J. Group Theory 6 (4) (2003), 461-476.

[9] C. Bates, D. Bundy, S. Perkins, P. Rowley: Commuting involution graphs in special linear groups, Comm. Algebra 32 (2004), no. 11, 4179-4196.

[10] C. Bates, D. Bundy, S. Perkins, P. Rowley: Commuting Involution Graphs for Sporadic Groups, J. Algebra 316(2007), 849 - 868. 
[11] C. Bates, D. Bundy, S. Perkins, P. Rowley. A Note on Commuting Graphs for Symmetric Groups, Elec. J. Combinatorics 16(2009), R6.

[12] B. Baumeister, A. Stein: Commuting graphs of odd prime order elements in simple groups, arXiv:0908.2583.

[13] D. Bundy: The connectivity of commuting graphs, J. Combin. Theory Ser. A, 113 (2006), no. 6, 995-1007.

[14] P.J. Cameron, S. Ghosh: The power graph of a finite group, Discrete Math. 311 (2011), no. 13, 1220-1222.

[15] R.W. Carter: Simple groups of Lie type, reprint of the 1972 original. Wiley Classics Library. A Wiley-Interscience Publication. John Wiley \& Sons, Inc., New York (1989). x+335 pp.

[16] W. Bosma, J. Cannon, C. Playoust: The Magma algebra system. I. The user language, J. Symbolic Comput., 24 (3-4) (1997), 235-265.

[17] A. Devillers, M. Giudici: Involution graphs where the product of two adjacent vertices has order three, J. Aust. Math. Soc. 85 (2008), no. 3, 305-322.

[18] A. Everett: Commuting Involution Graphs for 3-dimensional unitary groups, Electron. J. Combin. 18 (2011), no.1.

[19] A. Everett: Commuting Involution Graphs of Certain Finite Simple Classical Groups, PhD. Thesis, University of Manchester, 2011.

[20] B. Fischer: Finite groups generated by 3-transpositions, I. Invent. Math. 13 (1971), 232-246.

[21] D. Gorenstein: Finite groups, Second edition. Chelsea Publishing Co., New York (1980).

[22] S. Hoory, N. Linial, A. Wigderson: Expander graphs and their applications, Bull. Amer. Math. Soc. (N.S.) 43 (2006), no. 4, 439-561 (electronic).

[23] C. Jordan: Theoremes sur les groupes primitifs, J. Math. Pures Appl. 16 (1871), 383-408. 